\title{
Invited commentary for laparoscopic sleeve gastrectomy in patients with heart failure and left ventricular assist devices
} as a bridge to cardiac transplantation

\author{
Jeffrey E Friedman* \\ Medical Director of Metabolic and Bariatric Surgery, Department of Surgery, University of Florida, Gainesville, Florida, USA
}

\section{Commentary}

Obesity is a progressive, costly, genetically related, multi-factorial disease of excess fat storage. For many reasons, obesity rates have exponentially risen around the world. Morbid obesity affects every organ system in the body including the heart. The relationship between morbid obesity and heart failure is well established. Obesity causes both structural and functional changes in the heart, which can lead to left ventricular dilation, cardiac muscle hypertrophy, and atrial enlargement [1]. Heart failure has a very poor prognosis, with a mortality rate as high as $50 \%$ over 5 years [2]. Cardiac transplantation improves these patients 5 year survival to $80 \%$ and 10 year survival to $50 \%$ [3]. Patients with severe heart failure may require a left ventricular assist device (LVAD) for circulatory support. It is common for patients to gain weight after placement of the device due to improved quality of life. Weight loss is very difficult in this patient population due to exercise intolerance. The ultimate treatment for patients requiring LVAD support due to heart failure is cardiac transplantation. Most cardiac transplant centers use body mass index (BMI) of over $35 \mathrm{~kg} / \mathrm{m}^{2}$ as a contraindication to become eligible for transplantation [4].

In our retrospective study, which was conducted through the University of Florida's accredited bariatric center, patients who had severe end-stage heart failure due to noon-ischemic cardiomyopathy requiring support with a left ventricular assist device and a BMI greater than $35 \mathrm{~kg} / \mathrm{m}^{2}$ were referred to our bariatric surgery program. The University of Florida's bariatric program is certified through the metabolic and bariatric surgery accreditation and quality improvement program. Patients were referred to our program from the heart failure service with the goal of weight loss to reduce their BMI and become eligible for heart transplantation. A total of eleven patients who met these criteria completed the pre-operative program and subsequently underwent a laparoscopic sleeve gastrectomy (LSG). At the time of submission of this manuscript, seven $(64 \%)$ of our patients within the study reached a body mass index low enough to qualify for cardiac transplantation. Since publication, two more patients have achieved a BMI under 35 and are currently listed. The mean BMI a year following LSG was 33 and the mean percentage of excess weight loss was $60.9 \%$. The mean ejection fraction of these patients improved from $21.4 \%$ pre-operatively to $40.6 \%$ at 12 months post-operatively. Four of these patients underwent transplantation, while five of these patients are awaiting transplantation on the active list. One of the patients in this cohort died of an infection unrelated to surgery prior to transplant listing and one of the patients has yet to lose enough weight to qualify for listing. One patient, who lost over 100lbs, had significant improvement in ejection fraction from $15 \%$ pre-operatively to $65 \%$ at a year postoperatively. This patient is undergoing cardiac rehabilitation now with the goal of removing the LVAD.

\section{Conclusion}

Our study demonstrates that LSG is a safe surgical weight loss option for patients with morbid obesity and severe heart failure. A team approach utilizing the bariatric surgeons, cardiologists and anesthesiologist is critical for ensuring optimum results. This is an option for patients who require weight loss in order to be listed for cardiac transplantation.

\section{References}

1. Zhai, AB, Haddad H (2017) The impact of obesity on heart failure. Curr Opin Cardiol 32: 196-202. [Crossref]

2. Bui AL, Horwich TB, Fonarow GC (2011) Epidemiology and risk profile of heart failure. Nat Rev Cardiol 8: 30-41. [Crossref]

3. Lund LH, Khush KK, Cherikh WS, Goldfarb S, Kucheryavaya AY, et al. (2017) The Registry of the International Society for Heart and Lung Transplantation: Thirty-fourth Adult Heart Transplantation Report-2017; Focus Theme Allograft ischemic time. $J$ Heart Lung Transplant 36: 1037-1046. [Crossref]

4. Lee SJ, Kim KH, Hong SK, Hankins S (2017) Evaluation of a Heart Transplant Candidate. Curr Cardiol Rep 19: 133. [Crossref]
Copyright: (C)2018 Friedman JE. This is an open-access article distributed under the terms of the Creative Commons Attribution License, which permits unrestricted use, distribution, and reproduction in any medium, provided the original author and source are credited.
${ }^{\star}$ Correspondence to: Jeffrey E Friedman, Medical Director of Metabolic and Bariatric Surgery, Department of Surgery, University of Florida, Gainesville, Florida, USA, E-mail: Jeffrey.Friedman@surgery.ufl.edu

Received: July 24, 2018; Accepted: August 14, 2018; Published: August 17, 2018 